\title{
A numerical study on comparison of $1 D$ and 2D seismic responses of a basin in Turkey
}

\author{
Murat Emre Hasal ${ }^{1,}$, Recep Iyisan ${ }^{2}$ \\ ${ }^{1}$ Bursa Metropolitan Municipality, Department of Housing and Urban Development, Bursa, Turkey \\ ${ }^{2}$ Istanbul Technical University, Faculty of Civil Engineering, Civil Engineering Department, Istanbul, Turkey
}

\section{Email address:}

murat.hasal@bursa.bel.tr (M. E. Hasal), iyisan@itu.edu.tr (R. Iyisan)

\section{To cite this article:}

Murat Emre Hasal, Recep Iyisan. A Numerical Study on Comparison of 1D and 2D Seismic Responses of a Basin in Turkey. American Journal of Civil Engineering. Vol. 2, No. 5, 2014, pp. 123-133. doi: 10.11648/j.ajce.20140205.11

\begin{abstract}
Local site conditions such as seismic bedrock depth, bedrock slope of the edge, geometry and characteristics of soil layers, topographical irregularities, etc. are the most important factors affecting earthquake ground motion in a specific site. The amplitude and frequency content of bedrock motion can be changed by local site effects, and this variation is denoted as an amplification or de-amplification. Among the several factors, basin edge effect plays an important role in the transformation of earthquake waves and increase of the surface motion duration and amplitude. The limited width of the soil layers or the edge geometry at the deep formations cause earthquake wave transformations, thus the amplitude of the surface ground motion may vary depending on its location. For this reason the frequency content of surface ground motion may differ from the calculated surface ground motion by one dimensional dynamic analysis. In this case two dimensional analysis is required. In this study, in order to compare the soil response under different strong ground motion, one and two dimensional dynamic analyses were performed by using the Dinar Basin model in Turkey. The acceleration time histories and absolute acceleration spectra were obtained for pre-selected points on the ground surface. The 2D/1D spectral acceleration ratios were calculated by dividing the absolute acceleration spectra obtained from two dimensional (2D) and one dimensional (1D) dynamic analysis. The variations of the spectral acceleration ratios (2D/1D) with distance from basin edge were evaluated for different period values. The calculated $2 \mathrm{D} / 1 \mathrm{D}$ spectral acceleration ratios reached their maximum values at a certain zone $(\mathrm{X} / \mathrm{D}<3)$ near basin edge for every interested period value. While approaching to center of basin models, especially at the zones after $\mathrm{X} / \mathrm{D}=3$ point it can be noticed that $2 \mathrm{D} / 1 \mathrm{D}$ spectral acceleration ratios generally converged to 1 regardless of the edge bedrock slope values. The highest average spectral acceleration ratios were calculated when the relevant period values were between $\mathrm{T}=0.2 \sim 0.5 \mathrm{~s}$. They took values varying between 2 and 3 for this period interval. A relationship between the results of 1D and 2D dynamic analyses was established. In addition, the approximate validity range of $1 \mathrm{D}$ and 2D dynamic analysis at the basin edges was investigated for the model.
\end{abstract}

Keywords: 1D and 2D Dynamic Analysis, Amplification, Basin Edge Effect, 2D/1D Spectral Acceleration Ratio

\section{Introduction}

Evaluation of surface ground motion during earthquakes is one of the most important problems in geotechnical earthquake engineering. Severity and spatial distribution of surface motion during strong earthquakes is affected by geological and geotechnical conditions as well as earthquake source properties. The change in the characteristics of the earthquake such as amplitude, frequency content and duration due to geotechnical, geological and geometrical properties of a certain site can be defined as amplification. Local site conditions such as seismic bedrock depth, bedrock slope of the edge, geometry and characteristics of soil layers and topographical irregularities are the most important factors affecting soil amplification [1,2]. Topographical irregularities include both of the geological formations such as basins and valleys, the two and three dimensional geometry of subsurface soil layers and also the limiting bedrock boundaries. As a result, local site effects affect the damage variation occurred during the earthquakes and play an important role in the design of earthquake resistant structures. The purpose of the studies for local site effects is to determine the properties of earthquake design motion which is used for calculating the dynamic forces occurred at structures during earthquakes.

The difference of specific impedance between soil layers or soil layers and bedrock is the main reason for the changes in 
the characteristics of the seismic waves due to local site effects while propagating from bedrock to softer surface layers. One dimensional (1D) equivalent linear dynamic analysis method is the simplest approach to investigate local site effects which are generally complex because of the non-linear soil behavior. 1D dynamic analysis is usually preferred method because of its easiness in usage. It is based on the principle of vertically propagating body waves in the horizontally layered soil medium without any lateral boundaries [3].

The assumptions and boundary conditions of 1D approach become valid especially for the sites far from edges when the half-width of soil layers is much greater than its depth in shallow and wide basin models. However, in the case of the sites with topographical irregularities such as narrow valleys, basin edges, steep ridges and crests two and even three dimensional effects may occur [4]. These effects which bring up with the existence and interference of the body and surface waves can be studied by $2 \mathrm{D}$ and $3 \mathrm{D}$ numerical methods [5]. In order to understand the basic concepts of local site effects, the complex physical structure can be simplified as a horizontally layered one dimensional model, whereas the actual sedimentary deposits form mediums that can only be defined by $2 \mathrm{D}$ or $3 \mathrm{D}$ models. These real formations with lateral geological discontinuities show trapping behavior which gives rise to the surface waves and generally contribute them to reach their peak amplitude value. Also, the interference between the earthquake waves causes two and three dimensional resonance shapes to occur. The resonance modes seem simple in the case of one dimensional dynamic analysis; however they become more complex for 2D and 3D analyses. In the case of a shallow and broad basin/valley, the assumptions made for one dimensional behavior and boundary conditions become valid only for the middle part of the site; while for a deep and narrow basin/valley, since the back and forth reverberated waves are in the same phase with each other, the seismic waves form 2D resonance modes by interfering with each other [6,7]. For the conditions where two and three dimensional dynamic effects have to be considered, the amplitude and frequency content of surface motion will differ from the results of the 1D dynamic analysis calculated for the same site.

The change in frequency content and intensity of ground motion near basin edges can be defined as basin edge effect. This phenomenon depends mainly on the basin depth, edge slope and geometry, engineering properties of soil layers and characteristics of earthquake ground motion.

In this study, one and two dimensional dynamic analyses were performed by using the Dinar basin edge model to investigate the effects of basin edge on the variation of surface motion under different strong ground motion acceleration records and the results were compared. The variations of the spectral acceleration ratios (2D/1D) were evaluated for the different points on the ground surface with the changing distance from the basin edges.

Shear wave velocity profile and seismic bedrock depth and bedrock slope in Dinar basin were obtained by microtremor array measurements. The effect of edge bedrock slope on surface ground motion were investigated for four different slope values by performing 1D and 2D dynamic analyses on Dinar basin model. The acceleration time histories and absolute acceleration spectra were obtained for different points on basin surface. The 2D/1D spectral acceleration ratios were calculated by dividing the absolute acceleration spectra obtained from 2D and 1D dynamic analysis, and the change in $2 \mathrm{D} / 1 \mathrm{D}$ spectral acceleration ratio values with the distance from basin edge was investigated for different period values. A relationship depending on basin edge slope was suggested between absolute acceleration spectra obtained by $1 \mathrm{D}$ and 2D dynamic analyses in order to reflect the effects of 2D behavior at basin edge on 1D analysis. 1D and 2D dynamic analyses were performed by using Dyne-q [8] and Quake/W [9] softwares based on equivalent linear soil model, respectively. Dyne-q software operates in frequency domain and Quake/W software works in time domain.

\section{Dinar Basin}

Dinar is a town, which is located at the edge of an alluvial basin in southwest Anatolia, Turkey. On 1 October 1995, an earthquake of magnitude $\mathrm{M}_{\mathrm{L}}=5.9$ occurred in Dinar, Turkey. Noticeable structural damage was observed in a limited region located on the alluvium and adjacent to the rock outcrop bounding the eastern side of the town. In order to understand the effects of local site conditions to the variation of earthquake intensity throughout the town, numerous geotechnical studies including in-situ and laboratory experiments were conducted.

\subsection{General Geology and Tectonics of Region}

Quaternary alluvial sediments lay in the western part, Eocene limestone and sandstone exist in the northern regions and the geological formations at its eastern and southern regions are mainly composed of jura-cretaceous limestone and schist. The soil layers beneath the main settlement areas which are located in the center of Dinar town are composed of quaternary alluvial deposits and this area experienced high damage during the 1 October 1995 Dinar earthquake. During the drilling studies of water wells in Dinar basin, Oligocene conglomerate had been observed to underlie the alluvial sediments between $100 \mathrm{~m}-200 \mathrm{~m}$ depths [10]. The ground water table was located deeper than $20 \mathrm{~m}$ depth at the elevated sections of the town, but it was generally within a few meters at the flat sections of the basin.

There are two main fault systems around Dinar Town. One of them is Dinar-Civril fault system which lays in the NW-SE direction and the other is Akdag fault system with a direction of N-S. The Dinar-Civril fault, which was the source of seismic activity during the 1 October 1995 Dinar earthquake, is a $75 \mathrm{~km}$ long normal fault with a slight oblique left lateral component [11]. Those fault systems had caused a seismotectonic structure consisting of two grabens and a horst to occur. A characteristic geological section of Dinar lying in the E-W direction which reveals the horst-graben structure is illustrated in Fig. 1. 


\subsection{Local Soil Conditions}

Following the earthquake, extensive geotechnical investigations including numerous Standard Penetration Tests (SPT) in boreholes and cone penetration tests were conducted. The results of the field experiments indicated that nearly $80 \%$ of surface layers were mainly composed of medium stiff-stiff sandy clay and medium plasticity clay with thickness varying between 4-10 $\mathrm{m}$. The rest of the surface layers were composed of mostly medium dense and also loose clayey sand and gravel layers having thickness values between 1 4 $\mathrm{m}$ [10].

In two of the boreholes with approximate depths of $30 \mathrm{~m}$ PS
Logging tests had been performed to obtain the shear wave velocity profile of soil layers. After investigating the results of all of the mechanical and seismic field experiments carried out in Dinar region and comparing them carefully, it can be understood that the shear wave velocity takes values between $150 \mathrm{~m} / \mathrm{s}$ and $200 \mathrm{~m} / \mathrm{s}$ for the medium stiff clay layers, it is approximately $200 \mathrm{~m} / \mathrm{s}$ for the stiff clay layers and it approaches to values of $225 \mathrm{~m} / \mathrm{s}$ and $250 \mathrm{~m} / \mathrm{s}$ for the sandy stiff clay and gravelly stiff clay layers respectively. The pressure wave velocity took values around $1500 \mathrm{~m} / \mathrm{s}$ for the basin region generally indicating the shallow ground water level.
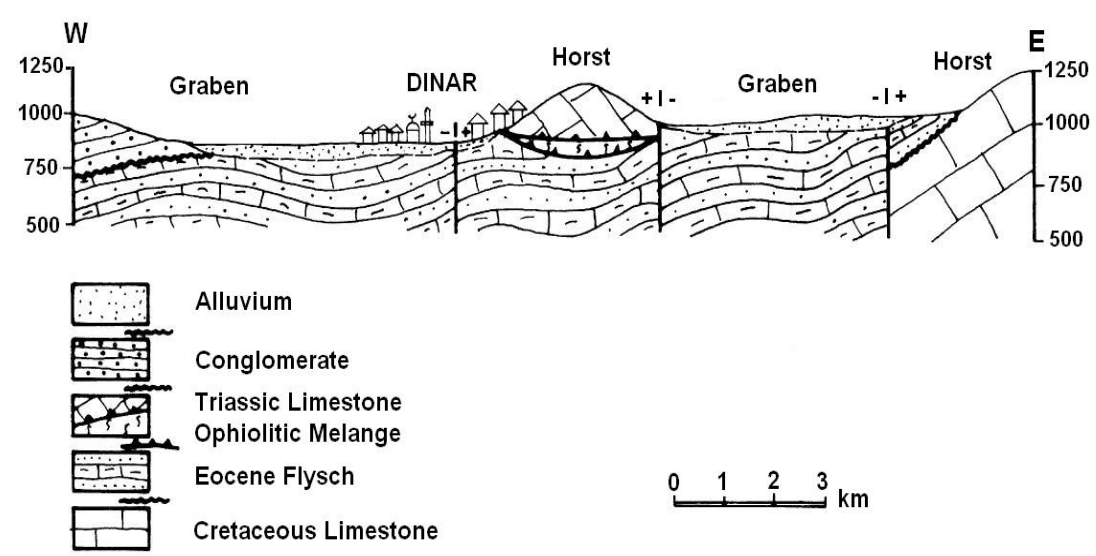

Figure 1. Geological cross section of Dinar region in E-W direction [10].

\subsection{Bedrock Depth from Microtremor Measurements}

As a well-known fact, determination of bedrock depth is very important for $2 \mathrm{D}$ dynamic analyses. The microtremor array measurements become very useful for estimating the seismic bedrock depth in case the boreholes are not deep enough to reach bedrock. The shear wave velocity profile at three different sections of Dinar basin was determined by microtremor array measurements. The data of microtremor array studies were combined with the topographical properties and geological section in Dinar to obtain two dimensional shear wave velocity profile and engineering properties of surface layers in the basin.

Single point microtremor measurements are widely used in geotechnical earthquake engineering to determine the local site predominant period, amplification and microzonation studies [12-18]. However, in order to determine the shear wave velocity profile from microtremor measurements and estimate the depth of seismic bedrock which can be defined as the media where the bedrock is rigid and its elastic properties remain nearly the same, the microtremors should be recorded continuously and simultaneously at multi-receiver instead of single point measurements [19]. The comparison of microtremors recorded in an array having a predetermined geometry and their frequency content, supply important data about dynamic properties of soil layers. This method is based on the determination of dispersion characteristics of surface waves.

In microtremor array measurements both of the receiver location geometry and the distance between each other may vary depending on the depth of soil layers. It is suggested that at least four receivers must be used in microtremor array measurements. An array consisting of receivers located at the corners and center of an equilateral triangle can be given as an example. In case there is no available space to form a triangle array geometry, receivers can be located linearly as well [19].

In order to determine the shear wave velocity profile and slope of edge bedrock in Dinar basin, microtremor array measurements were carried out at three different sites in Dinar as shown in Fig. 2. Microtremor data with sampling frequency of $100 \mathrm{~Hz}$ were simultaneously recorded by 4 receivers placed on the ground surface in predetermined distance. The array diameters were increased to obtain a precise phase velocity-period relation by keeping the center receiver fixed and removing the corner receivers far from the center and sufficient number of records was taken.

The most distant locations of the receivers used in the microtremor array measurements in Dinar were given in Fig. 2. The triangles in the map are symbolizing the center receivers for each of the three observations. At the beginning, the small array microtremors were recorded at all receivers for 15 minutes, then the receiver locations were changed four times to reach the large array by moving the corner receivers far from the center of triangle array. This process was also repeated at the other sites. The timing of the records was adjusted by the clock installed in the digitizer, so it became possible to compare the waveforms that were recorded at the same time interval. 


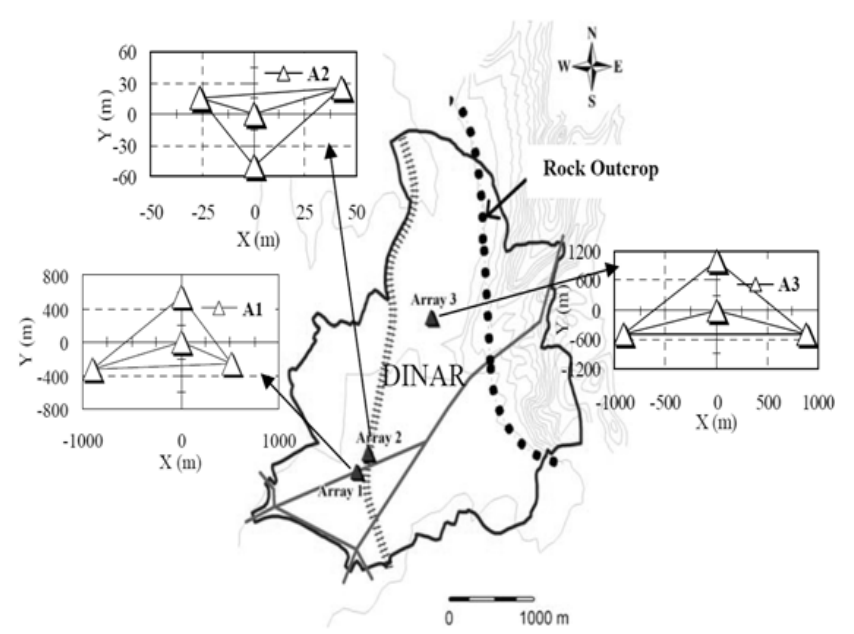

Figure 2. Microtremor array measurement locations in Dinar and array geometry.

\subsubsection{Estimation of Shear Wave Velocity Profile}

In microtremor observations Rayleigh waves composed of vertical components of surface waves are mainly recorded. Rayleigh waves are dispersive, in other words the velocity of Rayleigh waves $\left(V_{R}\right)$ varies depending on the frequency ( $f$ ) and wavelength $(\lambda)$ values $\left(V_{R}=\lambda f\right)$. The velocity of seismic waves for a specific frequency value can be defined as phase velocity (c). The graphics show that the variation of phase velocity with frequency or period values is called as dispersion curve. This curve can be obtained theoretically for a given soil profile and it is a function of the shear wave velocity $\left(\mathrm{V}_{\mathrm{s}}\right)$, pressure wave velocity $\left(\mathrm{V}_{\mathrm{p}}\right)$, unit weight $(\gamma)$ and thickness of soil layers. As a result, the phase velocity values and their variation with frequency can be used to estimate the dynamic characteristics of the soil layers where the array measurements were carried out $[19,20]$. With this aim, the microtremors that were recorded with the receivers in an array are transferred into frequency domain from time domain by spectral analysis technique. This process can be easily executed with the help of Fast Fourier Transformation (FFT) method. After this, the cross-power spectra between microtremor waveforms such as $\mathrm{x}(\mathrm{t})$ and $\mathrm{y}(\mathrm{t})$ that were recorded at two neighbor receivers having predetermined coordinates is calculated with the help of FFT technique by using the relation given below [21].

$$
\mathrm{CPS}_{\mathrm{x}, \mathrm{y}}(\mathrm{f})=\mathrm{LS}_{\mathrm{x}}(\mathrm{f}) \cdot \mathrm{LS}_{\mathrm{y}}^{*}(\mathrm{f})
$$

In this equation, $\operatorname{LS}_{\mathrm{x}}(\mathrm{f})$ is the linear spectrum of microtremor record $\mathrm{x}(t)$ (or its Fourier spectrum), $\mathrm{LS}_{\mathrm{y}} *(\mathrm{f})$ is the complex conjugate of linear spectrum of $y(t)$ and $f$ is the frequency. This function gives the phase difference between two wave records for each frequency $\left(\theta_{\mathrm{x}, \mathrm{y}}\right)$, thus the interval time, $t_{I(f)}$, for a microtremor wave between two considered receivers is calculated with (2).

$$
\mathrm{t}_{\mathrm{I}(\mathrm{f})}=\frac{\theta_{\mathrm{x}, \mathrm{y}}(\mathrm{f})}{360 . \mathrm{f}}=\frac{\theta_{\mathrm{x}, \mathrm{y}}(\mathrm{f}) \mathrm{T}}{360}
$$

As it is known, phase difference value of $360^{\circ}$ is equal to arrival time of one period. Frequency-wave number (f-k) spectral analysis is the simplest theoretical method used to obtain the spreading velocity of microtremor waves at different frequencies. f-k spectrum is a Fourier transformation shows the plane and direction of wave propagation. The $f-k$ spectrum of recorded waves at any two receivers can be given as below [21].

$$
P(f, k)=\sum_{x, y=i}^{n} \operatorname{CPS}_{x, y} \cdot e^{i k\left(X_{x}-X_{y}\right)}
$$

In (3), $X_{x}$ ve $X_{y}$ are the receiver coordinates, $n$ is the number of receivers and $\mathrm{k}$ shows the vector of wave number with a dimension of $1 / \mathrm{km}$. Phase velocity, c, can be calculated as below for the greatest value of $\mathrm{f}-\mathrm{k}$ spectrum.

$$
\mathrm{c}=\frac{2 \pi \mathrm{f}}{\mathrm{k}}=\frac{\omega}{\mathrm{k}}
$$

In this equation, $\omega$ is circular frequency. This calculation process is repeated for every frequency, so dispersion curves can be obtained and the dynamic characteristics of the soil layers can be estimated by using inversion theory. In order to obtain acceptable values for dynamic characteristics, the theoretical values calculated by analyzing a selected soil profile with assigned soil properties must be similar to observed values. The dispersion curve of a layered soil profile is controlled mainly by shear wave velocity $\left(\mathrm{V}_{\mathrm{s}}\right)$ and thickness (D) of layers. As a result of the array observations carried out at three different sites of Dinar, the vertical microtremor records were analyzed by the method which was briefly mentioned above, thus their dispersion curves were obtained and the relevant soil properties were estimated by using inversion theory [22]. In order to succeed these process, model parameters of the theoretical dispersion curve calculated for four layered soil profile, was changed until the theoretical dispersion curve became very close to observed one from microtremor measurements. The dispersion curve obtained from the first microtremor array measurements performed with a dimension of $1000 \mathrm{~m}$ and the variation of shear wave velocity profiles which were estimated for all of the three array measurements by using inversion method with theoretical soil properties, were shown together in Fig. 3.

As it can be understood from Fig. 3, the seismic bedrock $(\mathrm{Vs} \approx 1000 \mathrm{~m} / \mathrm{s}$ ) was reached at $175 \mathrm{~m}$ depth for the first microtremor array measurements which had been performed at the basin center; however it was reached at $85 \mathrm{~m}$ and $68 \mathrm{~m}$ depths for the other sites respectively. These results pointed out that seismic bedrock at basin edge was laying in NE-SW direction with a slope value of 1/10 [23]. The layer thickness and shear wave velocity values obtained at three microtremor array measurement sites were used to constitute the two dimensional model of Dinar basin. 

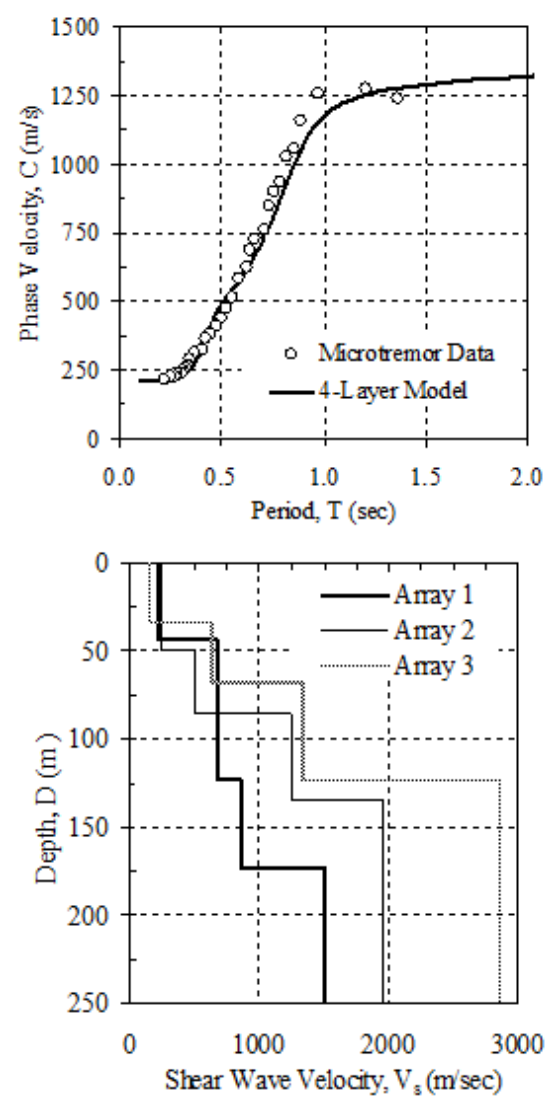

Figure 3. Field dispersion curves and the variation of shear wave velocity with depth.

\subsubsection{Basin Model}

Bedrock slope at basin edge, the frequency content of bedrock earthquake motion, depth and width of basin affect the dynamic behavior of soil layers during strong earthquakes. In order to investigate the effect of basin edge slope on surface motion, Dinar basin model was set up initially and dynamic analyses were done for models with four different basin edge slope values. When geological section of Dinar and extensive field studies carried out in the basin were taken into consideration with the shear wave velocity profile obtained from microtremor array observations, it was estimated that bedrock at basin edge was dipping towards basin center with a slope value of $1 / 10\left(6^{\circ}\right)$. In the basin, sandy and silty low-intermediate plasticity clay layers were laying above bedrock consecutively. In order to obtain the properties of near surface soil layers, the results of former in-situ and laboratory test results were used $[23,26]$. In this study, initially the effect seismic bedrock accelerogram of 1 October 1995 Dinar Earthquake on Dinar basin model was investigated. The acceleration time history of bedrock motion had been obtained by deconvolution the surface accelerograms to seismic bedrock at $180 \mathrm{~m}$ depth. After this, it was aimed to consider the second dimension and edge effect on basin model generally, thus in order to achieve this, the changes in the dynamic behavior of the basin model was tried to be evaluated by using different edge slope values and bedrock accelerograms. With this aim, 1D and 2D dynamic analyses were executed for 12 different sites at basin edge by using 4 different edge slope values $\left(\alpha=6^{\circ}, 11^{\circ}, 27^{\circ}, 45^{\circ}\right)$ as given in Table 1 and results of the analyses were compared.

Table 1. The geometrical properties of Dinar Basin.

\begin{tabular}{lllll}
\hline Model No. & $\mathbf{D}(\mathbf{m})$ & $\mathbf{H}(\mathbf{m})$ & H/D & $\boldsymbol{\alpha}\left({ }^{\circ}\right)$ \\
\hline 1 & 180 & 1800 & 10 & 6 \\
2 & 180 & 900 & 5 & 11 \\
3 & 180 & 360 & 2 & 27 \\
4 & 180 & 180 & 1 & 45 \\
\hline
\end{tabular}

The finite element mesh of Dinar basin model which was used in dynamic analyses and its boundary conditions are illustrated in Fig. 4. In Fig. 4; D shows bedrock depth, $\mathrm{H}$ is basin edge width and $\mathrm{X}$ can be defined as the distance from edge bedrock outcrop to basin center. In the analyses, the soil profile over bedrock was divided into 18 different layers with $10 \mathrm{~m}$ thickness for each and shear wave velocity values of 200 $\mathrm{m} / \mathrm{sec}$ and $1000 \mathrm{~m} / \mathrm{sec}$ were assigned to uppermost layer and seismic bedrock respectively.

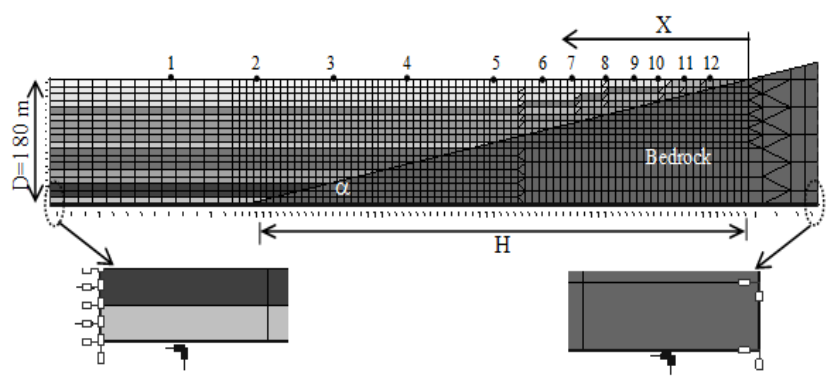

Figure 4. The boundary conditions and finite element mesh of Dinar Basin model used in dynamic analyses.

The soil layers above seismic bedrock were assumed to be composed of intermediate plasticity clay $(\mathrm{CI}, \mathrm{Ip}=20 \sim 25 \%)$. The curves representing the change in damping ratio (D) and shear modulus $(\mathrm{G})$ of the soil layers with cyclic shear strain $\left(\gamma_{\text {cyc }}\right)$ were constituted by using Ishibashi-Zhang [24] relation. Also it was assumed that the transition zone between rigid bedrock and soil layers was composed of weathered rocks, so the change in damping ratio values with cyclic shear strain and stiffness degradation for this zone was modeled with the relation proposed by Schnabel et al. [25]. The stiffness degradation curve of the soil layers and the change in damping ratio with cyclic shear strain is illustrated in Fig. 5.

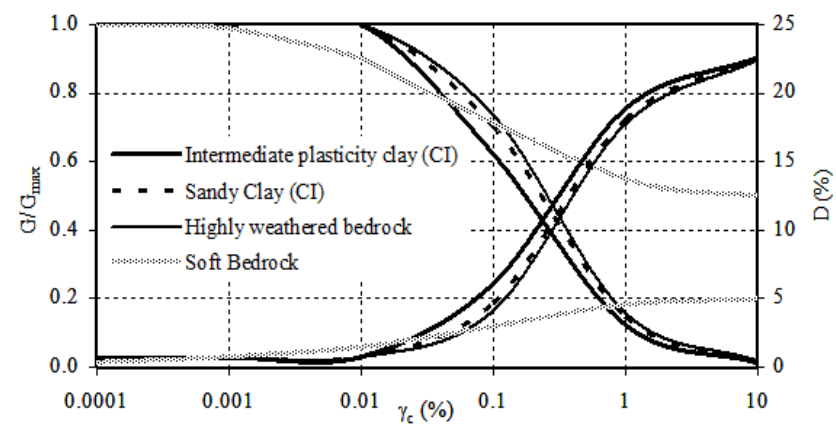

Figure 5. Stiffness degradation and damping ratio curves for soil layers in Dinar basin. 


\section{Characteristics of Strong Ground Motion Used in the Study}

In order to reflect the characteristics of bedrock motion to the dynamic behavior of soil layers, four different acceleration time histories with different intensity parameters and frequency content were used in the dynamic analyses. Two of those accelerograms belonged to Turkey earthquakes and the others were from strong ground motion records of San Andreas Fault system that has similar characteristics with North Anatolian Fault.

Table 2. The general characteristics of strong ground motion acceleration records used in the study.

\begin{tabular}{|c|c|c|c|c|}
\hline Earthquake Name & Palm Springs (1986) & Dinar (1995) & Mendocino (1992) & Kocaeli (1999) \\
\hline Station & Silent Valley & Meteorological Agency & Cape Petrolia & Sakarya Pub. \\
\hline Formation & Weathered Granite & Deconvolution Analysis & Rock & Sandstone \\
\hline Magnitude & $\mathrm{M}_{\mathrm{L}}=5.9$ & $\mathrm{M}_{\mathrm{L}}=5.9$ & $\mathrm{M}_{\mathrm{L}}=5.9$ & $\mathrm{M}_{\mathrm{d}}=7.4$ \\
\hline Depth (km) & 11.1 & 12.0 & 14.6 & 18.0 \\
\hline Distance $(\mathrm{km})$ & 19.5 & 2.0 & 15.0 & 35.0 \\
\hline $\mathrm{a}_{\max }(\mathrm{g})$ & 0.1 & 0.2 & 0.3 & 0.4 \\
\hline
\end{tabular}
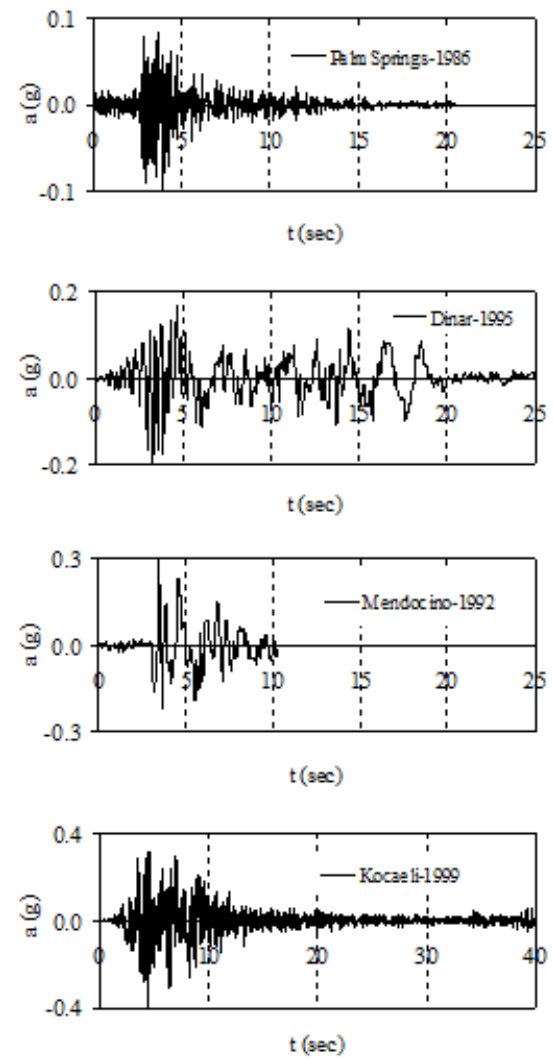

Figure 6. The strong ground motion accelerograms used in the analyses.

One of the accelerograms used in the dynamic analyses was obtained by using the E-W component of Dinar Meteorology Station acceleration time history which had been recorded at 1 October 1995 Dinar Earthquake. In the region where Dinar Meteorology Station was located, microtremor array measurements had been carried out and it was found out that seismic bedrock lies at great depth from the surface. The recorded surface ground motion of E-W component of Dinar Earthquake was deconvoluted to bedrock by 1D dynamic analysis by using the soil profile obtained from the extensive field and laboratory studies done in the region [26]. This accelerogram was used as a bedrock motion in the dynamic analyses. The other strong ground motion which belonged to Turkey earthquake excitations was recorded at the building of Sakarya Public Works and Settlement Directorate on 17 August 1999 Kocaeli earthquake. Strong ground motion of 1986 Palm Spring and 1992 Mendocino earthquakes were recorded at San Andreas Fault system.

The acceleration time histories of earthquake excitations used in the analyses were illustrated in Fig. 6. These accelerograms were band-pass filtered between $0.10-25 \mathrm{~Hz}$ and baseline corrections were done. Then, their peak accelerations were scaled to the nearest values among $0.1 \mathrm{~g}$, $0.2 \mathrm{~g}, \quad 0.3 \mathrm{~g}$ and $0.4 \mathrm{~g}$ [23]. The properties of selected accelerograms used in this study were given in Table 2 .

\section{Methods Used in Dynamic Analyses}

The numerical methods developed for the evaluation of dynamic behavior of soil layers against the earthquake excitation are defined as 1D, 2D and 3D with respect to the requirements of the problem considered. 1D numerical method is mainly preferred because of the simplicity of its theoretical background and easiness in usage. On the contrary, in 2D dynamic analysis methods; 2D geometry, shear wave velocity profile and boundary conditions are required for setting up the model and more knowledge is needed to make comments on the results obtained. In this study, Dyne-q [8] and Quake/W [9] softwares that are based on the equivalent linear method were used in 1D and 2D dynamic analyses, respectively.

\subsection{Method for 1D Dynamic Analysis}

In this study; 1D dynamic analysis software, Dyne-q [8] was used in order to determine 1D dynamic response of soil layers against the earthquake excitations. Dyne-q [8] is a computer code for the earthquake response analysis of level ground, which is based on the equivalent linear method and multiple reflection theory. In 1D approximation, the ground motion in any layer can be estimated by using one 
dimensional wave propagation theory in layered media. Briefly in this method, the surface motion is obtained by the inversion process of the Fourier series of the ground surface (output) motion which can be defined as the product of the transfer function of layered soil deposits and the Fourier series of bedrock (input) motion. In this study; the material properties such as soil type, thickness, unit weight and shear wave velocity were obtained from the geotechnical investigations, but the variation in the damping ratio (D) and shear modulus $(\mathrm{G})$ of the soil layers with cyclic shear strain $\left(\gamma_{c}\right)$ was empirically modeled by using Ishibashi-Zhang [24] relation. It was assumed that soft bedrock was lying at the transition zone between soil layers and medium hard bedrock. The variation of the dynamic characteristics with cyclic shear strain for this zone was defined by using the relation of Schnabel et al. [25]. The stiffness degradation curve of the soil layers and the change in damping ratio with cyclic shear strain is depicted in Fig. 5.

\subsection{Method for 2D Dynamic Analysis}

2D dynamic analyses are generally performed by using numerical methods such as finite difference, finite element or hybrid methods. The finite difference method generally uses a uniform mesh for modeling the seismic wave propagation in an elastic media. It is simple and easy to apply but insufficient to simulate complex boundary conditions such as surface topography, subsurface geometry and sloping bedrock. The finite element method allows irregular mesh with elements having different sizes and geometries to be used therefore it is very useful for modeling complex geometry and boundary conditions [27]. Quake/W software [9] that is based on the equivalent linear method, was used in 2D dynamic analyses in this study. The numerical code of this software has a finite element approach in which the governing motion equation for dynamic response of a system can be expressed as:

$$
[\mathrm{M}]\{\ddot{\mathrm{u}}\}+[\mathrm{C}]\{\dot{\mathrm{u}}\}+[\mathrm{K}]\{\mathrm{u}\}=\{\mathrm{F}\}
$$

Where; $[\mathrm{M}]$ is mass matrix, $[\mathrm{C}]$ is damping matrix, $[\mathrm{K}]$ is stiffness matrix, $\{\mathrm{F}\}$ is vector of loads, $\{\ddot{\mathrm{u}}\}$ is nodal acceleration vector, $\{\dot{\mathrm{u}}\}$ is nodal velocity vector, $\{\mathrm{u}\}$ is nodal displacement vector. Damping term is usually defined as a linear combination of mass $(\mathrm{m})$ and rigidity $(\mathrm{k})$ as below.

$$
\begin{gathered}
\mathrm{c}=\mathrm{a}_{0} \mathrm{~m}+\mathrm{a}_{1} \mathrm{k} \\
\mathrm{a}_{0}=\xi\left(\left(2 \omega_{\mathrm{i}} \omega_{\mathrm{j}}\right) /\left(\omega_{\mathrm{i}}+\omega_{\mathrm{j}}\right)\right) \text { and } \mathrm{a}_{1}=\left(2 \xi /\left(\omega_{\mathrm{i}}+\omega_{\mathrm{j}}\right)\right) \\
\left.\xi=\left(\mathrm{a}_{0} / 2\right)\left(1 / \omega_{\mathrm{n}}\right)+\left(\mathrm{a}_{1} / 2\right) \omega_{\mathrm{n}}\right)
\end{gathered}
$$

In the equations given above, $\mathrm{a}_{\mathrm{o}}$ and $\mathrm{a}_{1}$ are scalars and expressed as Rayleigh damping coefficients. $\xi$ is the damping ratio of a system for the $\mathrm{n}^{\text {th }}$ mode, $\omega_{\mathrm{n}}$ is natural circular frequency, $\omega_{\mathrm{i}}$ and $\omega_{\mathrm{j}}$ can be expressed as the natural circular terms corresponding to $i^{\text {th }}$ and $\mathrm{j}^{\text {th }}$ modes of the system. Lysmer and Richart [28] proved that the amplitudes of the body waves consisting of pressure $(\mathrm{P})$ and shear $(\mathrm{S})$ waves can be diminished for different incoming angles if absorbent boundaries are used in the $2 \mathrm{D}$ dynamic analyses. The damping terms used for absorbing $\mathrm{P}$ and $\mathrm{S}$ waves can be defined as;

$$
\mathrm{c}_{\mathrm{p}}=\rho \mathrm{V}_{\mathrm{p}} \text { and } \mathrm{c}_{\mathrm{s}}=\rho \mathrm{V}_{\mathrm{s}}
$$

Here $c_{p}$ and $c_{s}$ are damping coefficients that are used to absorb the energy of $\mathrm{P}$ and $\mathrm{S}$ waves. $\mathrm{V}_{\mathrm{p}}$ and $\mathrm{V}_{\mathrm{s}}$ are $\mathrm{P}$ and $\mathrm{S}$ wave velocity of the relevant soil layers and $\rho$ is the density of layers. The absorbent effect of soil layers and bedrock lying at the vertical and horizontal boundaries of the finite element model can be taken into consideration by putting viscous dashpots. In case the base of the model is fixed with restraints in both directions and especially when studied with strong ground motion acceleration which cause nonlinear behavior of the soil layers, the soil amplifications at the surface layers may reach to unrealistic high values during the numerical analyses. For this reason, viscous dashpots in two directions should be put at the base boundary of the model. Dashpot coefficients are proportional with the pressure and shear wave values of the relevant soil layers at the boundaries of 2D model. In addition to the dashpots, the effect of the 1D free field motion was added to the model by applying time dependent stress functions at both of the vertical boundaries. Those boundary forces were calculated by multiplying the 1D particle velocity values of the soil layers with the relevant horizontal dashpot coefficients and applied to the 2D model at the boundaries as stress functions changing throughout the earthquake ground motion.

In the equivalent linear method, the maximum value of normalized nodal displacements $\left(\mathrm{U}_{\max }\right)$ are calculated during the dynamic analysis and compared with the values obtained from preceding iteration. During the execution of dynamic analysis by equivalent linear method, the calculations will continue until reaching the user defined iteration number or the difference between normalized displacements falls under a predetermined convergence $\left(\delta_{\mathrm{T}}\right)$ value as defined below. $\mathrm{U}_{\max (\mathrm{i})}$ is the normalized maximum displacement at $\mathrm{i}^{\text {th }}$ iteration.

$$
\delta \mathrm{U}_{\max }=\left(\mathrm{ABS}\left(\mathrm{U}_{\max (\mathrm{i}+1)}-\mathrm{U}_{\max (\mathrm{i})}\right) / \mathrm{U}_{\max (\mathrm{i})}\right)<\delta_{\mathrm{T}}
$$

\section{Results of 2D Dynamic Analyses}

In two dimensional dynamic analyses, Quake/W finite element software [9], which is based on the principle of equivalent linear method, was used. After the 2D analyses, the maximum absolute horizontal acceleration values obtained at the surface $\left(a_{\text {max }_{-} s}\right)$ for 12 different points of the model as shown in Fig. 4, were normalized by the peak horizontal acceleration at rock outcrop $\left(\mathrm{a}_{\text {max_r}}\right)$. These normalized values were defined as soil amplification $\left(\mathrm{a}_{\max -\mathrm{s}} /\right.$ $\left.\mathrm{a}_{\max \_}\right)$. The variation of the soil amplifications calculated for different earthquakes with bedrock slope and X/D dimensionless distance was given in Fig. 7. 

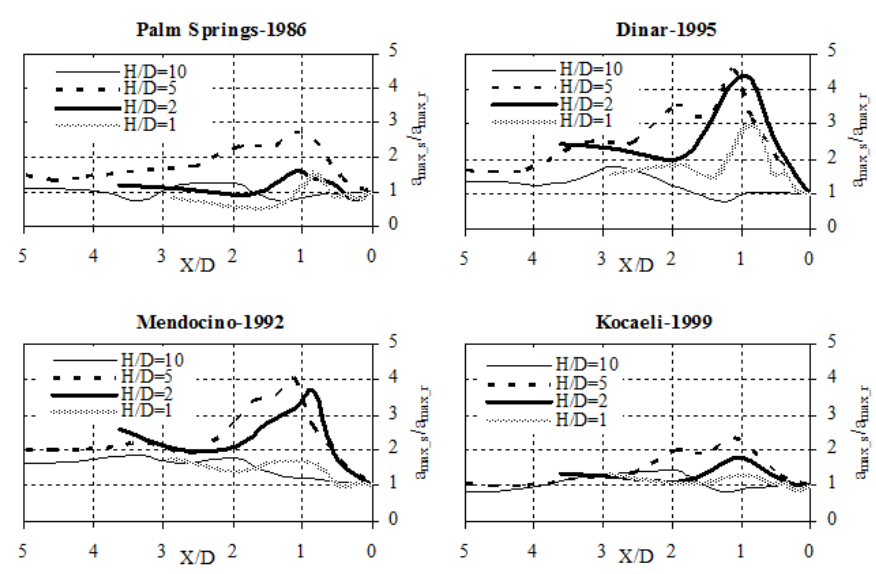

Figure 7. Soil amplifications calculated for different earthquakes and bedrock slope values.

Acceleration spectrum intensity, ASI, which had been proposed to define the behavior of rigid structures with predominant periods lower than $0.5 \mathrm{sec}$ under strong ground motion is given as below [29]. In the equation $\mathrm{S}_{\mathrm{a}}$ and $\xi$ show acceleration spectrum and damping ratio respectively and $\mathrm{T}$ is period value. ASI values can be related to the behavior of rigid structures with predominant periods lower than $0.5 \mathrm{sec}$.

$$
\mathrm{ASI}=\int_{0.1}^{0.5} \mathrm{~S}_{\mathrm{a}}(\xi=0.05, \mathrm{~T}) \mathrm{dT}
$$

The change in ASI values with the distance from edge outcrop to middle sections of basin are illustrated in Fig. 8 for models with different edge bedrock slope values. As it can be seen from Fig. 7 and 8, soil amplification and ASI values reach to their peak values at a definite edge section while moving away from rock outcrop to basin center and afterwards, with the increase in X/D value they converge to each other for every earthquake excitation without depending on the bedrock slope value.
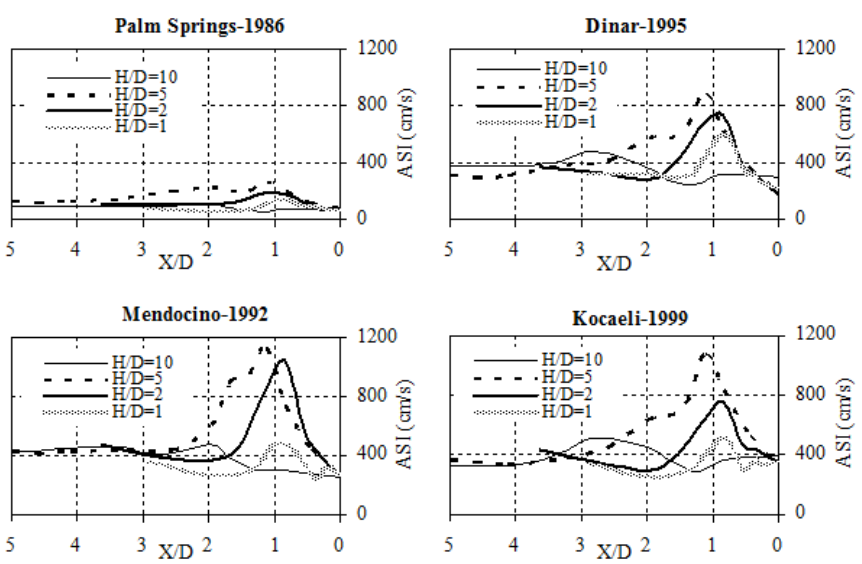

Figure 8. ASI values calculated for different earthquake and bedrock slope.

\section{Comparison of 1D and 2D Dynamic Analyses}

In order to estimate the surface ground motion at basin edges with different bedrock slope, 2D finite element method which is based on equivalent linear method was used in the dynamic analyses for different earthquake excitations and the findings were compared with the results of $1 \mathrm{D}$ dynamic analysis. With this purpose, absolute acceleration spectra were obtained for the different sections of all models by using the acceleration time histories obtained from 1D and 2D dynamic analyses. Dyne-q software [8] that had been developed basing on modified equivalent linear method was used to execute $1 \mathrm{D}$ dynamic analysis.

The difference between 2D and 1D dynamic behaviors was evaluated by proportioning the acceleration spectra that were calculated by $2 \mathrm{D}$ and $1 \mathrm{D}$ analyses respectively. The ratio of the acceleration spectra that are obtained as a result of $2 \mathrm{D}$ and 1D dynamic analyses is defined as "aggravation factor" [30]. In order to investigate the effect of surface ground motion on structures with different rigidity or period values, 2D/1D spectral acceleration ratios were calculated for 5 different period values ( $\mathrm{T}=0,0.3,0.4,0.6,0.9 \mathrm{~s})$ by using different basin edge geometry and earthquake excitations. The relevant 2D/1D spectral acceleration ratio (aggravation factor) curves are shown in Fig. 9 for the case of $\mathrm{T}=0.3 \mathrm{~s}$.
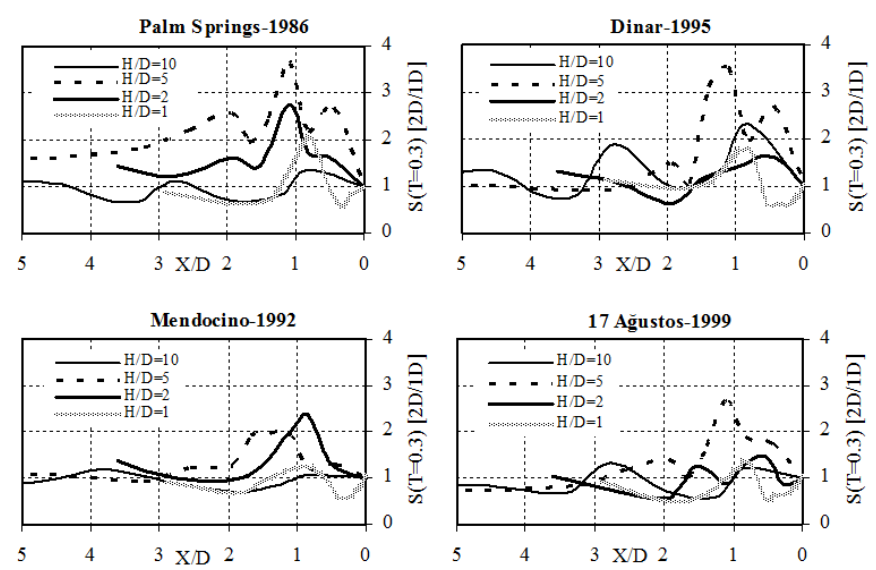

Figure 9. The variation of $2 D / 1 D$ spectral acceleration ratios with $X / D$.

2D/1D spectral acceleration ratios reached to their peak values at a certain edge section and afterwards, while moving away from rock outcrop to basin center; spectral acceleration ratios approximately converged to 1 for each period. Also it can be realized that 2D/1D spectral acceleration ratios approached to each other after a definite value of $\mathrm{X} / \mathrm{D}$ $(\mathrm{X} / \mathrm{D}=3)$ regardless of the edge bedrock slope value. Spectral acceleration ratios took values between $2 \sim 4$ depending on the edge bedrock slope and they reached to their peak values when H/D is equal to $5\left(\alpha=11^{\circ}\right)$.

Without depending on the period values, average 2D/1D spectral acceleration ratio values converged to 1 after the points of $\mathrm{X} / \mathrm{D}=5,4,2$ and 1.5 when the edge bedrock slope values were $\alpha=6^{\circ}, 11^{\circ}, 27^{\circ}$ and $45^{\circ}$ respectively. After those, $2 \mathrm{D}$ effects were much reduced. By benefiting from these results, the limits of the sections at basin edges where 2D dynamic behavior under earthquake excitation should be taken into account can be obtained. In Fig. 10, the validity limits for 1D and 2D dynamic analysis are depicted for 
related models depending on varying values of basin geometry.
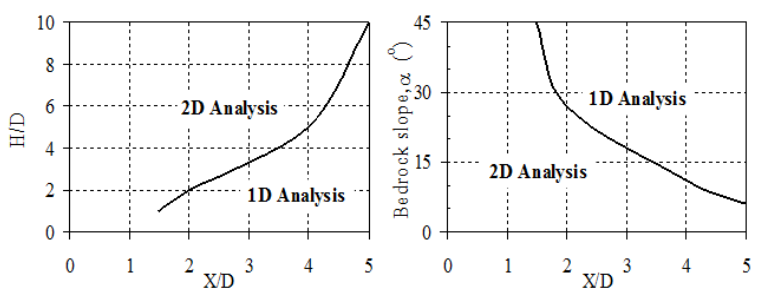

Figure 10. The variation of the dynamic approach effectiveness with the edge geometry at Dinar basin models.

\section{Results and Discussion}

The acceleration spectra were obtained for different points at a basin surface as a result of $1 \mathrm{D}$ and 2D dynamic analyses which had been performed on basin models with different edge bedrock slope values. 2D/1D spectral acceleration ratios were calculated for 5 different period values in order to investigate the relation between spectral acceleration values that had been obtained by $1 \mathrm{D}$ and 2D dynamic analyses and to estimate the effect of 2D geometry on the spectral acceleration values calculated from 1D dynamic analyses. 2D/1D spectral acceleration ratio values were assumed to be normally distributed for different earthquake excitations and the variation of average ratios with both of X/D and period values were illustrated as 3D surfaces in Fig. 11 for 4 different bedrock slope values. As it can be seen from the Fig. $11,2 \mathrm{D} / 1 \mathrm{D}$ spectral acceleration ratios reached to their peak values for the model with $H / D=5$. The $2 \mathrm{D} / 1 \mathrm{D}$ spectral acceleration ratios especially decreased for the models with lower bedrock slope values. The highest spectral acceleration ratio values were obtained at the period interval of $0.2 \sim 0.5 \mathrm{~s}$ for all basin models. As the edge bedrock slope value decreased, the difference between spectral acceleration values decreased too.
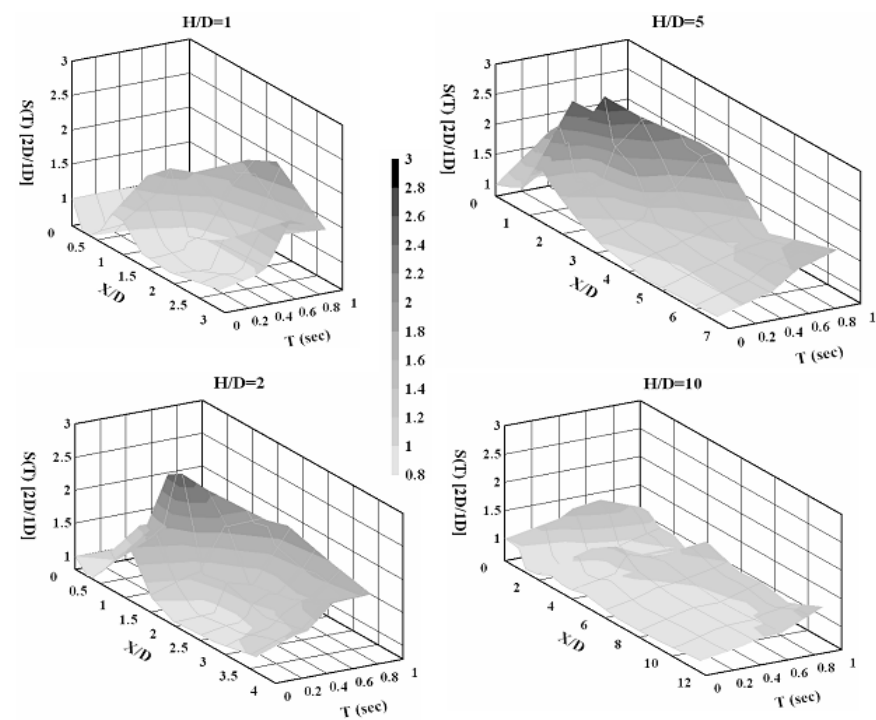

Figure 11. The variation of $2 D / 1 D$ spectral acceleration ratios with $X / D$ and period values.
In the dynamic analyses done, the variation of $2 \mathrm{D} / 1 \mathrm{D}$ spectral acceleration ratios with $\mathrm{X} / \mathrm{D}$ dimensionless parameter were investigated for basin edge. With this purpose, the average of spectral acceleration ratios at different periods were calculated and after evaluating the results, the relation given below was obtained on condition that $\mathrm{X} / \mathrm{D}>0$.

$$
S(T)\left[\frac{2 D}{1 D}\right]=e^{\left\{a+\frac{b}{(X / D)}+c \ln \left(\frac{X}{D}\right)\right\}}+1
$$

In this relation; the aggravation factor is expressed by $\mathrm{S}(\mathrm{T})[2 \mathrm{D} / 1 \mathrm{D}] . \mathrm{X}$ is the distance from rock outcrop, $\mathrm{D}$ is the depth to seismic bedrock, all of $\mathrm{a}, \mathrm{b}$ and $\mathrm{c}$ show the coefficients that are dependent on edge bedrock slope and period values. The values of these coefficients depend on each other and for the model with $\mathrm{H} / \mathrm{D}=5$, where spectral acceleration ratios reach their highest values, they took values of $14,-14$ and -10 respectively. Equation (12) was very successful at estimating the variation of 2D/1D spectral acceleration ratio values with $\mathrm{X} / \mathrm{D}$ however it could not model the behavior of some aggravation factor curves which had more than one maximum and minimum value. However, it is thought that the behavior of sudden increase and decrease in aggravation factor values that is recognized close to bedrock outcrop at edges of some models can not come into existence perfectly. The reason for this consideration is generally the existence of soil layers at basin edges that are stiffer or denser than sedimentary formations lying at middle parts of basin surface. Those stiffer soil layers can be classified as talus and residual soil which were formed by accumulation of rock fragments at the base of cliffs and by chemical/physical weathering of native bedrock in place respectively. For this reason, the small scaled secondary increments and decrements in the values of aggravation factors for the zones of alluvium located near the edge bedrock outcrop were neglected.

\section{Conclusions}

In this study, the effect of basin edge slope on surface ground motion was tried to be estimated by performing $1 \mathrm{D}$ and 2D dynamic analyses on Dinar basin model for 4 different slope values. With this aim; acceleration time histories, acceleration spectra, intensity parameters were obtained for basin surface and the variation of these values with the distance from edge bedrock outcrop were investigated for different bedrock earthquake excitations. In order to determine the difference between the results of one and two dimensional dynamic analyses, the acceleration spectra which were calculated for different sections of basin by using 2D dynamic analyses were divided by the ones calculated with $1 \mathrm{D}$ dynamic analyses, so that $2 \mathrm{D} / 1 \mathrm{D}$ acceleration spectrum ratios were obtained. After this, the change in 2D/1D spectral acceleration ratio values with the distance from edge rock outcrop was studied and a relationship between the 
acceleration spectra of 1D and 2D dynamic analyses was tried to be established. The depth and edge bedrock slope value for two dimensional models were obtained from the shear wave velocity profile calculated from microtremor array measurements.

The maximum increments in horizontal acceleration and acceleration spectrum intensity-ASI values, which were calculated as a result of the dynamic analyses done on models with varying edge bedrock slope values by using different earthquake bedrock excitations, appeared between the beginning of edge bedrock outcrop and $\mathrm{X} / \mathrm{D}=3$ point, especially for the edge bedrock slope angle values of $11^{\circ}$ and $27^{\circ}(\mathrm{H} / \mathrm{D}=5$ and 2$)$ in comparison with other models.

The calculated 2D/1D spectral acceleration ratios reached their maximum values at a certain zone $(X / D<3)$ near basin edge for every interested period value. At this zone, average aggravation factors took values between 0.5 and 4.0 for different strong ground motions. While approaching to center of basin models, especially at the zones after $X / D=3$ point it can be noticed that 2D/1D spectral acceleration ratios generally converged to 1 regardless of the edge bedrock slope values. At these sections, $1 \mathrm{D}$ and $2 \mathrm{D}$ dynamic analyses give similar results. For all the basin models with different edge bedrock slope values, the maximum average aggravation factors were relatively obtained for the edge bedrock slope angle value of $11^{\circ}(\mathrm{H} / \mathrm{D}=5)$. With the decrease in edge bedrock slope value $\left(H / D=10, \alpha=6^{\circ}\right)$, the difference between $1 \mathrm{D}$ and 2D spectral acceleration values became negligible. For all the models, the highest average spectral acceleration ratios were calculated when the relevant period values were between $\mathrm{T}=0.2 \sim 0.5 \mathrm{~s}$. The average spectral acceleration ratio values vary between 2 and 3 for this period interval. By using the 2D/1D aggravation factor-spectral acceleration ratio relation obtained in this study, it will be possible to reflect the second dimension effect to the spectral acceleration values calculated from 1D dynamic analysis depending on edge bedrock slope value and $\mathrm{X} / \mathrm{D}$ term.

This relation was obtained for the case of Dinar basin model with varying edge bedrock slope values. It is expected to be useful for estimating the effect of second dimension on the spectral acceleration values obtained for basin edge sections from 1D dynamic analyses. Also it will be possible to develop and change the relevant relation with the help of future research results.

\section{References}

[1] G. A. Athanasopoulos, P. C. Pelekis, and E. A. Leonidou, "Effects of surface topography on seismic ground response in the Egion (Greece) 15 June 1995 Earthquake", Soil Dynamics and Earthquake Engineering, vol. 18, pp. 135-149, 1999.

[2] E. Safak, "Local site effects and dynamic soil behaviour", Soil Dynamics and Earthquake Engineering, vol. 21, pp. 453-458, 2001.

[3] S. L. Kramer, Geotechnical Earthquake Engineering, Prentice-Hall, Upper Saddle River, USA, 1996.
[4] K. Pitilakis, Recent Advances in Earthquake Geotechnical Engineering and Microzonation, A. Ansal (ed.), Kluwer Academic Publishers, Netherlands, pp. 139-193, 2004.

[5] R. Iyisan and H. Khanbabazadeh, "A numerical study on the basin edge effects on soil amplification", Bulletin of Earthquake Engineering, vol. 11, Issue 5, pp. 1305-1323, 2013.

[6] M. Rassem, A. Ghobarah, and A. C. Heidebrecht, "Engineering perspective for the seismic site response of alluvial valleys", Earthquake Engineering and Structural Dynamics, vol. 26, pp. 477-493, 1997.

[7] R. Iyisan and M. E. Hasal, "The effect of ground motion characteristics to the dynamic response of alluvial valley models", $13^{\text {th }}$ Asian Regional Conference of Soil \& Geotechnical Engineering, Theme-7 Dam Engineering, Paper Code 7.1-8, Kolkata, India, 2007.

[8] DYNEQ, A Computer Program for Dynamic Response Analysis of Level Ground By Equivalent Linear Method, Version 3.23, Revised from Original Version (May 1995) by Yoshida and Suetomi., 2003.

[9] QUAKE/W, Finite Element Dynamic Earthquake Analysis Software, Geo-Slope Office.

[10] B. S. Bakir, M. Y. Ozkan, and S. Ciliz, "Effects of basin edge on the distribution of damage in 1995 Dinar, Turkey Earthquake", Soil Dynamics and Earthquake Engineering, vol. 22, pp. 335-345, 2002.

[11] A. Ozturk, "Tectonics of Dinar Sandıkl1-Isıklı region", Communications of Faculty of Science, University of Ankara, No.25, pp. 1-58, 1982.

[12] M. E. Hasal, R. Iyisan, H. Khanbabazadeh, A. Bayin, G. Cevikbilen, and O. Kepceoglu, "A preliminary seismic microzonation study based on microtremor measurements", International Conference on Earthquake Engineering, Skopje, Macedonia, 2013.

[13] R. Iyisan, M. E. Hasal, G. Cevikbilen, and A. Bayin, "Estimation of site response by microtremor measurements", 2013 International Van Earthquake Symposium, Abstract ID:100, Van, Turkey, 2013.

[14] A. J. Choobbasti, S. Rezaei, and F. Farrokhzad, "Evaluation of site response characteristics using microtremors", Gradevinar, 65, pp. 731-741, 2013.

[15] S. Rezaei, A. J. Choobbasti, and S. S. Kutanaei, "Site effect assessment using microtremor measurement, equivalent linear method, and artificial neural network (case study: Babol, Iran)", Arabian Journal of Geosciences, DOI 10.1007/s12517-013-1201-1, 2013.

[16] M. K. Kockar and H. Akgun, "Evaluation of the site effects of the Ankara basin, Turkey", Journal of Applied Geophysics, vol. 83, pp. 120-134, 2012.

[17] P. Teves-Costa and P. Y. Bard, "Seismic behaviour estimation of thin alluvium layers using microtremor recordings", Soil Dynamics and Earthquake Engineering, vol. 15, pp. 201-209, 1996.

[18] R. Iyisan and A. Ansal, "Estimation of local soil conditions with microtremor", $7^{\text {th }}$ National Congress on Soil Mechanics and Foundation Engineering (in Turkish), YTU, İstanbul, pp. 542-549, 1998. 
[19] R. Iyisan, U. Gulerce, H. Yamanaka, and A. Ansal, "Estimation of shear wave velocity by microtremor observations", $9^{\text {th }}$ National Congress on Soil Mechanics and Foundation Engineering (in Turkish), Anadolu Üniversitesi, Eskişehir, 2002.

[20] H. Yamanaka, "Geophysical exploration of sedimentary structures and their characterization", The Effects of Surface Geology on Seismic Motion, Balkema, Rotterdam, pp.15-33, 1998.

[21] M. Horike, "Geophysical exploration using microtremor measurements", Proc. of $11^{\text {th }}$ World Conf. on Earthquake Engineering, paper no. 2033, 1996.

[22] H. Yamanaka and H. Ishida, "Application of genetic algorithms to an inversion of surface-wave dispersion data", Bulletin of the Seism. Society of America, vol. 86, No.2, pp.436-444, 1996.

[23] M. E. Hasal, The Effects of Topographical Irregularities on Soil Amplification, Ph. D. Thesis (in Turkish), ITU Institute of Science and Technology, İstanbul, 2008.

[24] I. Ishibashi and X. Zhang, "Unified dynamic shear moduli and damping ratios of sand and clay", Japanese Society of Soil Mechanics and Foundation Eng., vol. 33, No.1, pp. 182-191, 1993.

[25] P. B. Schnabel, J. Lysmer, and H. B. Seed, Shake: A Computer
Program for Earthquake Response Analysis of Horizontally Layered Sites, Report No. UCB/EERC 72-12, Earthq. Eng. Research Center, University of California, Berkeley, p. 102, 1972.

[26] M. A. Ansal, R. Iyisan, and H. Gullu, "Microtremor measurements for the microzonation of Dinar", Pure and Applied Geophysics, vol. 158, pp. 2525-2541, 2001.

[27] B. Gatmiri, C. Arson, and K.V. Nguyen, "Seismic site effects by an optimized 2D BE/FE method: Theory, numerical optimization and application to topographical irregularities", Soil Dynamics and Earthquake Engineering, vol. 28, pp. 632-645, 2008.

[28] J. Lysmer and R. L. Kuhlemeyer, "A finite dynamic model for infinite media", Journal of the Engineering Mechanics Division, ASCE, vol. 95, No. EM4, pp. 859-877, August, 1969.

[29] J. L. Von Thun, L. H. Rochim, G. A. Scott and J. A. Wilson, "Earthquake ground motions for design and analysis of dams", Earthquake Engineering and Soil Dynamics II, Recent Advance in Ground-Motion Evaluation, Geotec. Special Publication, vol. 20, ASCE, New York, pp. 463-481, 1988.

[30] K. Makra, D. Raptakis, F. J. Chavez-Garcia, and K. Pitilakis, "Site effects and design provisions: The case of Euroseistest", Journal of Pure and Applied Geophysics, vol. 158, N.12, pp. 2349-2367, 2001 